\title{
ANÁLISE LOGÍSTICA DA MATRIZ FERROVIÁRIA NACIONAL ATRAVÉS DE PESQUISA OPERACIONAL
}

\author{
K. J. Faria ${ }^{1}$; J. V. S. M. Catarino ${ }^{2}$; D. F. Vicentini ${ }^{3}$ \\ ${ }_{1,2,3}$ Universidade Federal do Paraná. Av. Coronel Francisco H. dos Santos, 210. Jd. das Américas, Curitiba/PR \\ CEP 81530-000 \\ kaio.faria@hotmail.com ${ }^{1}$,joao.catarino@ufpr.br $r^{2}$,vicentini@ufpr.br ${ }^{3}$
}

\begin{abstract}
Resumo: A malha ferroviária brasileira possui grande importância no escoamento da produção nacional, mesmo sendo subutilizada em termos proporcionais, ao se comparar, por exemplo, o uso de ferrovias em outros países com características similares ao Brasil. Além da subutilização do potencial ferroviário do país, ainda existem diversos pontos passíveis de melhoria da malha nacional, em termos de logística, otimização de processos e operaçôes. Neste estudo, a malha ferroviária brasileira é analisada com um olhar voltado para a logística do sistema nacional como uma análise a nível macro. O objetivo deste trabalho foi estudar a malha, detectar gargalos e deficiências operacionais e propor melhorias ao sistema em termos de custo, eficiência, reduçáo do tempo de transporte, entre outros. Para isso foi desenvolvida uma ferramenta de Pesquisa Operacional que permitiu estudar a malha, visando encontrar gargalos operacionais no sistema, além de possíveis oportunidades de melhoria, como redução de custos, melhoria de eficiência, diminuição de tempo de transporte, entre outros. $\mathrm{O}$ estudo indicou pontos de déficit no escoamento de cargas para os portos brasileiros, identificando-se trechos para maior destinação de recursos.
\end{abstract}

Palavras-chave: Ferrovias, Logística, Pesquisa operacional.

Abstract: The Brazilian rail network has great importance in the flow of domestic production, even though it is underused in proportional terms, when comparing, for example, the use of railroads in other countries with similar characteristics to Brazil. Besides the underutilization of the country's rail potential, there are still several possible points of improvement of the national network in terms of logistics and optimization of processes and operations. The main purpose of this work was to apply Operational Research tools, aiming at finding operational difficulties in the system, as well as possible opportunities for improvement, such as cost reduction, efficiency improvement, and shorter transport time, among others. The study indicated points of deficit in the flow to the Brazilian ports, identifying segments for the greater allocation of resources.

Keywords: Railways, Logistics, Operational research. 


\section{Introduçáo}

O transporte ferroviário no Brasil apresenta relevante importância econômica, porém possui diversos gargalos e ineficiências, o que pode gerar um baixo aproveitamento de todo o potencial ferroviário que o país possui. Em [1] é relatado que as estradas de ferro são fontes quase inesgotáveis de problemas de planejamento, e até recentemente todos os problemas eram tratados manualmente, sendo que muitos ainda são resolvidos sem nenhuma automação ou otimização. Embora o modal ferroviário apresente importante papel no país, seu potencial de utilização ainda não foi inteiramente desenvolvido.

Segundo dados encontrados em [2], no Brasil em 2015, a produção ferroviária atingiu 332 bilhóes de TKU (toneladas por quilômetro útil), o que significa um aumento de 142\% desde o início das concessôes. No mesmo período (entre 1997 e 2015), o PIB brasileiro cresceu 53,1\% e atingiu R \$ 5,9 trilhóes em 2015. Isso significa que a taxa de aumento da produção ferroviária é quase o triplo do crescimento do PIB brasileiro.

Dada a importância do transporte ferroviário para a economia do país, esta pesquisa pretende propor melhorias ao sistema ferroviário nacional, buscando otimizar sua utilizaçáo e melhorar a eficiência geral do sistema. A área em questão apresenta muitas necessidades de melhoria, e também possui um grande potencial de utilização e crescimento. Ao propor soluçôes que otimizem processos dentro do setor, podem-se trazer diversos benefícios ao país, tanto econômicos quanto sociais.

Este estudo apresenta um panorama geral do modal, apresentando um mapa em forma de rede de nós de parte da malha ferroviária brasileira, considerando seus principais pontos, produtos transportados, além de parâmetros importantes como o custo, para poder propor melhorias em possíveis gargalos encontrados, fazendo uso de conhecimentos de programação linear e pesquisa operacional.

\section{Metodologia}

Inicialmente foi feito um levantamento dos dados, o que permitiu a análise sob um panorama logístico, onde foi possível aplicar ferramentas de Pesquisa Operacional a fim de observar as possíveis alternativas para a melhoria das malhas ferroviárias. Assim, a partir do estudo inicial da malha, foi possível concluir que os quatro principais materiais transportados pela rede ferroviária são: Minério de Ferro, Soja, Produção Agrícola (principalmente Açúcar e Milho) e Combustíveis. As interaçóes resultaram da manipulação e observância do conjunto de pontos, denominados nós, estes ligados por linhas, definindo os arcos, conjuntos que compóem o grafo, conforme descreve [7] e representado pela Fig. 1. Analisando o fluxo entre os nós do grafo, é possível determinar as redes, sendo estas subdivididas de acordo com caraterísticas específicas, visando o objeto de estudo. 


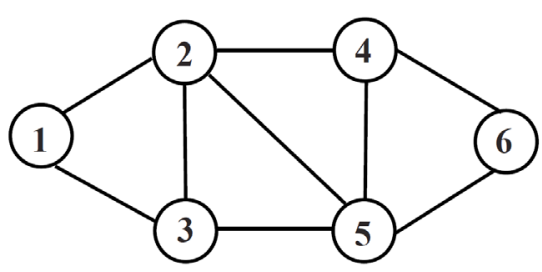

Figura 1 - Exemplo de rede.

Fonte: [7].

Um método de solução para problemas de Programação Linear foi indicado por [5], criando o Simplex. Este método tem por função determinar valores para as variáveis a fim de satisfazer um conjunto de equaçóes lineares, indicando o objetivo de minimizar ou maximizar uma forma $\mathrm{Z}$ definida como resultado da matriz.

Dentre as várias otimizaçóes interessadas no tangente à resolução de equaçóes de Programação Linear, é possível observar características comuns dentre as situaçóes. Para tanto, [2] definem, dentre as características, variáveis manipuláveis indicando o poder de decisão sobre o problema; valores que devem compor uma regiấo de soluçóes em que se apliquem as consideraçóes, ainda variáveis capazes de assumir valores previamente determinados dentre os números reais.

A aplicabilidade do método Simplex foi possibilitada pela utilizaçáo da ferramenta Solver, possibilitando-se encontrar um caminho mínimo da rede. Esta ferramenta está presente em programas de organização de dados, tais como o Microsoft Excel, sendo apresentada nas opçóes no campo de suplementos no programa. O Solver permite que se encontrem soluçóes viáveis para as matrizes elaboradas baseadas nas redes das malhas ferroviárias com a utilização do Simplex para então propiciar inserção no estudo logístico das ferrovias.

Buscando a aplicação dos conhecimentos junto ao programa Excel, algumas consideraçóes foram tomadas para possibilitar essa execução. Assim sendo, primeiramente foram definidos os nós como variáveis $\mathrm{X}_{i}$ e posteriormente as possíveis trajetórias a serem seguidas no desenvolvimento do algoritmo foram identificadas. Isso permitiu que cada arco fosse tratado com a indicação das iniciais de cada nó, os quais se conectavam, tendo por variável $\mathrm{X}_{i j}$.

As nomenclaturas para cada um dos pontos da rede são apresentadas na imagem a seguir: 
Tabela 1 - Nomenclatura utilizada na matriz aplicada.

\begin{tabular}{|ll|ll|}
\hline Anápolis & $\mathrm{X}_{\mathrm{a}}$ & Rondonópolis & $\mathrm{X}_{\mathrm{ro}}$ \\
Roncador & $\mathrm{X}_{\mathrm{r}}$ & Aparecida do Taboado & $\mathrm{X}_{\mathrm{ap}}$ \\
Brasília & $\mathrm{X}_{\mathrm{b}}$ & Araraquara & $\mathrm{X}_{\mathrm{ara}}$ \\
Araguari & $\mathrm{X}_{\mathrm{ar}}$ & Colombia & $\mathrm{X}_{\mathrm{co}}$ \\
Uberaba & $\mathrm{X}_{\mathrm{u}}$ & Bauru & $\mathrm{X}_{\mathrm{ba}}$ \\
Belo Horizonte & $\mathrm{X}_{\mathrm{bh}}$ & Itirapina & $\mathrm{X}_{\mathrm{iti}}$ \\
Jaceaba & $\mathrm{X}_{\mathrm{j}}$ & Alumínio & $\mathrm{X}_{\mathrm{al}}$ \\
Saudade & $\mathrm{X}_{\mathrm{s}}$ & Japeri & $\mathrm{X}_{\mathrm{ja}}$ \\
Joaquim Murtinhc & $\mathrm{X}_{\mathrm{jm}}$ & Itaguai & $\mathrm{X}_{\mathrm{ita}}$ \\
Barra do Pirai & $\mathrm{X}_{\mathrm{bp}}$ & Rio de Janeiro & $\mathrm{X}_{\mathrm{rj}}$ \\
Boa Vista & $\mathrm{X}_{\mathrm{bv}}$ & Montes Claros & $\mathrm{X}_{\mathrm{mc}}$ \\
Jundiaí & $\mathrm{X}_{\mathrm{jn}}$ & Camaçari & $\mathrm{X}_{\mathrm{ca}}$ \\
São Paulo & $\mathrm{X}_{\mathrm{sp}}$ & Aracaju & $\mathrm{X}_{\mathrm{aj}}$ \\
Santos & $\mathrm{X}_{\mathrm{sn}}$ & Vitoria & $\mathrm{X}_{\mathrm{vi}}$ \\
\hline
\end{tabular}

Fonte: Os autores (2017)

Em seguida, devido às especificaçóes para a aplicabilidade do problema de pesquisa operacional apresentado por [7], foram identificadas as variáveis de restrição. Estas foram evidenciadas pela observação da interligação dos nós apresentados pelas variáveis já indicadas, onde 1 foi atribuído para caminhos com variáveis interligadas aos nós e 0 para variáveis que não estấo conectadas. A Fig. 2 apresenta um exemplo aplicável das matrizes encontradas.

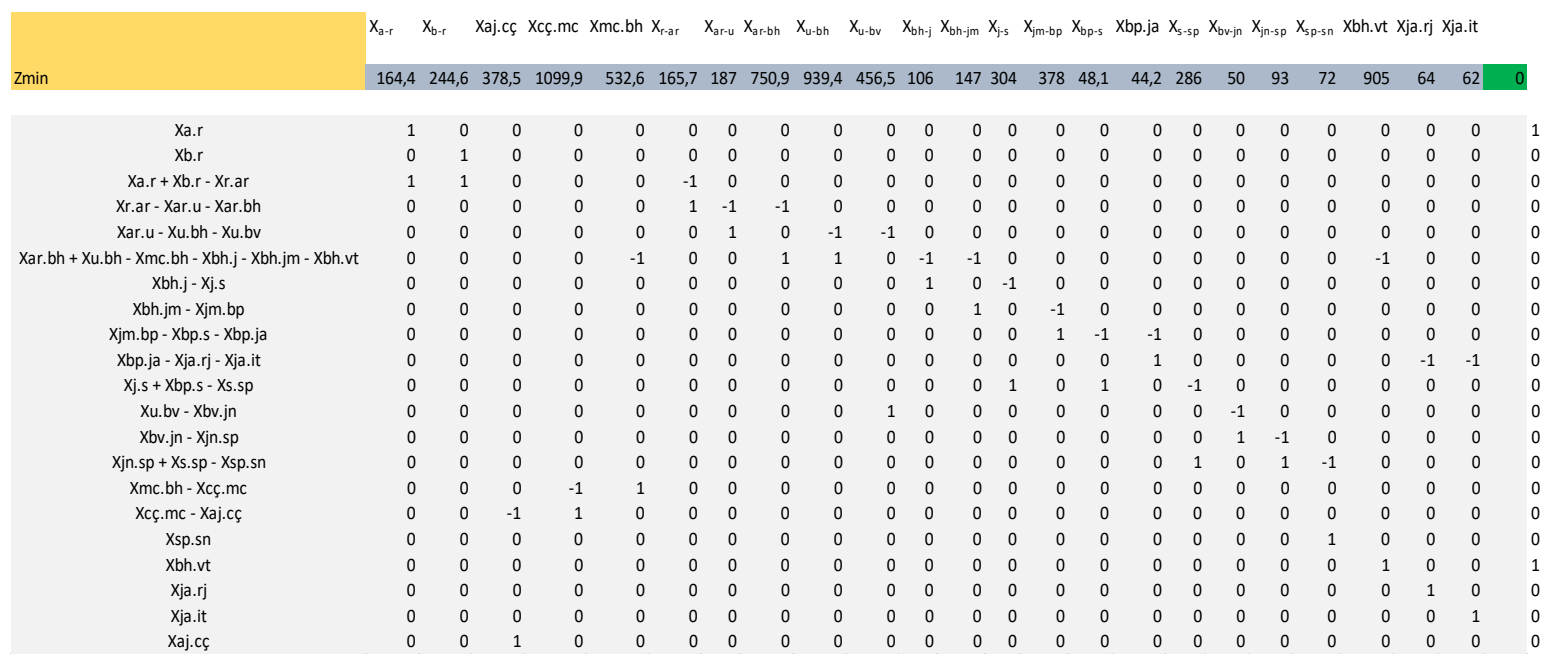

Figura 2 - Matriz de interaçóes para resolução com ferramenta Solver.

Fonte: Os autores (2017) 


\section{Resultados}

Para aplicar o estudo do Caminho Mínimo, foi necessário criar uma rede de nós que envolvesse pontos relevantes para a malha nacional, e que estivessem interligados, possibilitando, assim, aplicar a metodologia, visando encontrar informaçóes relevantes quanto à distância e tempo de transporte necessários para cada origem e destino.

Como resultado, foram obtidas as malhas tais como a apresentada na Fig. 3 (rede de combustíveis), que envolve cinco trechos concessionados, principalmente na região Sudeste. Com essa malha, foi possível aplicar pesquisa operacional com diversas opçôes de origens e destinos.

Conforme apresentado na seção de metodologia, foi utilizada a ferramenta Solver, do Microsoft Excel para aplicar diversas interaçóes com todas as possíveis origens e destinos aplicáveis à malha. Abaixo, a matriz de aplicação criada é apresentada, onde apenas a origem e o destino foram alterados em cada interação, bem como a tabela com resultados de distância e tempo para cada um dos cenários analisados.

$\mathrm{Na}$ Tabela 2 apresentam-se as variáveis de restrição aplicadas, em que todas as possíveis origens e todos possíveis destinos são representados pelo número 1 . As variáveis apresentadas representam o arco que corresponde à interligação das cidades, tomando-se como exemplo temos $\mathrm{X}_{\mathrm{a} . \mathrm{r}}$ representando o trecho de conexão entre Anápolis - Roncador Novo, seguindo-se consequentemente para os outros pontos.

Tabela 2 - Variáveis de restrição da rede.

\begin{tabular}{|c|c|c|c|}
\hline \multicolumn{3}{|c|}{ Variáveis de Restrição } & 1 \\
\hline Xa.r & 1 & Xro.ap & 0 \\
\hline Xb.r & 1 & Xro.ap-Xap.ara & 1 \\
\hline Xa.r + Xb.r - Xr.ar & 0 & Xco.ara & 0 \\
\hline Xr.ar - Xar.u - Xar.bh & 0 & Xap.ara+Xco.ara-Xara.iti & 1 \\
\hline Xar.u - Xu.bh - Xu.bv & 0 & Xba.iti & 0 \\
\hline Xar.bh + Xu.bh - Xbh.j - Xbh.jm - Xbh.mc- Xbh.vi & 0 & Xba.iti + Xara.iti - Xiti.bv & 0 \\
\hline Xbh.j - Xj.s & 0 & Xbv.al - Xal.sn & 0 \\
\hline Xbh.jm - Xjm.bp & 0 & Xbp.ja - Xja.ita - Xja.rj & 1 \\
\hline Xjm.bp - Xbp.s - Xbp.ja & 0 & Xja.ita & 1 \\
\hline Xj.s +Xbp.s - Xs.sp & 0 & Xja.rj & 0 \\
\hline Xu.bv + Xiti.bv - Xbv.jn - Xbv.al & 0 & Xbh.mc - Xmc.ca & 0 \\
\hline Xbv.jn - Xjn.sp & 0 & Xmc.ca - Xca.aj & 1 \\
\hline Xjn.sp + Xs.sp - Xsp.sn & 0 & Xca.aj & 1 \\
\hline Xsp.sn+Xal.sn & 1 & Xbh.vi & 1 \\
\hline
\end{tabular}

Fonte: Os autores (2017). 


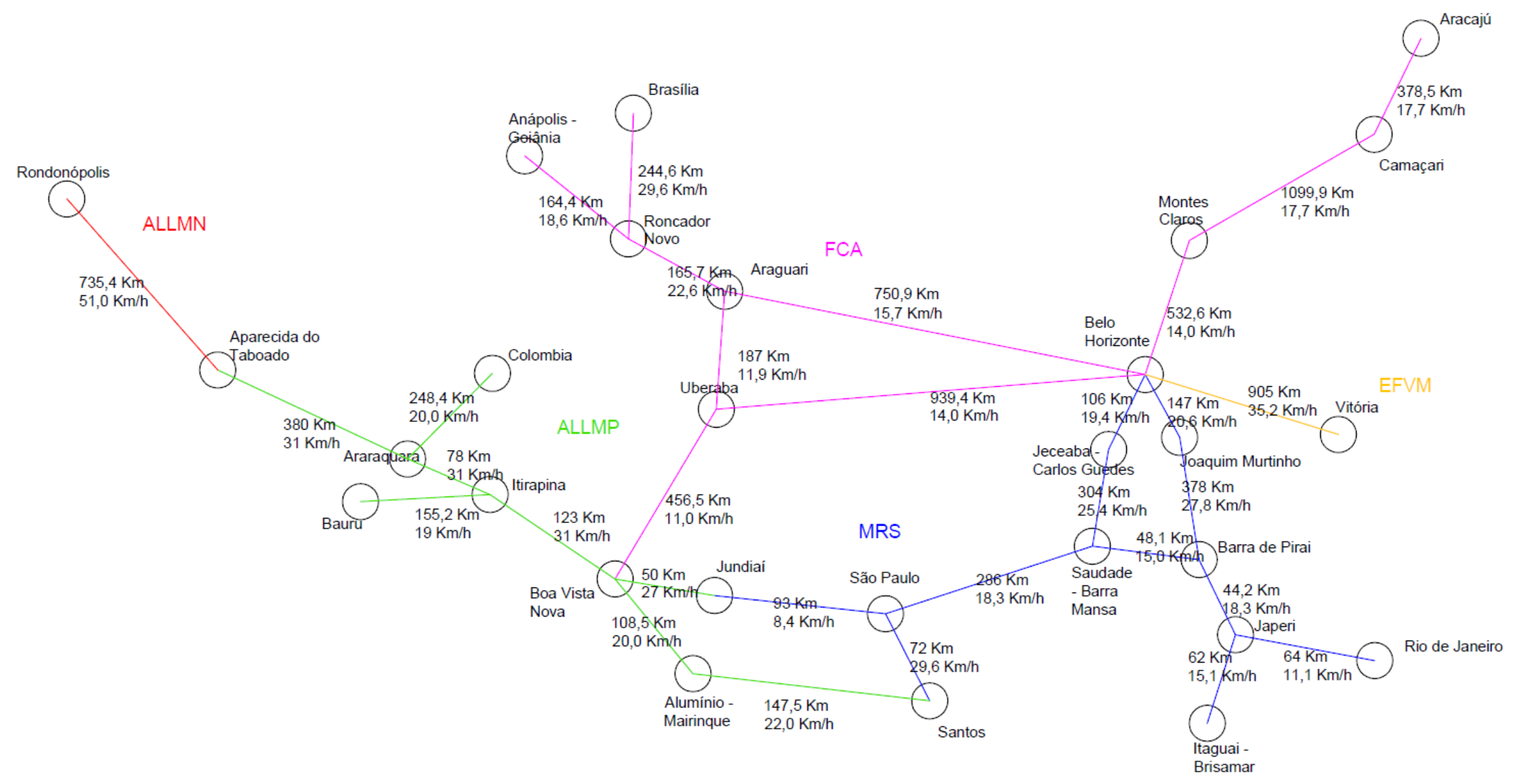

Figura 3 - Rede de nós da malha ferroviária (rede combustíveis).

Fonte: Os autores (2017). 
A malha apresentava quatro possíveis origens: Anápolis (representada por $\mathrm{X}_{\mathrm{a}}$ ), Brasília (representada por $\left.\mathrm{X}_{\mathrm{b}}\right)$, Rondonópolis $\left(\mathrm{X}_{\mathrm{ro}}\right)$ e Bauru $\left(\mathrm{X}_{\mathrm{ba}}\right)$. Todas essas origens poderiam chegar em cinco possíveis destinos: Santos $\left(\mathrm{X}_{\mathrm{sn}}\right)$, Itaguaí $\left(\mathrm{X}_{\mathrm{ita}}\right)$, Rio de Janeiro $\left(\mathrm{X}_{\mathrm{rj}}\right)$, Vitória $\left(\mathrm{X}_{\mathrm{ri}}\right)$ e Aracaju $\left(\mathrm{X}_{\mathrm{aj}}\right)$, gerando, no total, vinte interaçóes, com os resultados apresentados na Tabela 3.

Tais resultados também podem justificar o motivo para a grande utilização do modal rodoviário ao invés do ferroviário para transporte do produto. A velocidade média dos trechos estudados, para o modal ferroviário segundo esta pesquisa, foi entre 15 e $20 \mathrm{Km} / \mathrm{h}$, o que pode influenciar na decisão para realizar o transporte via rodovias. Sabe-se que, apesar do transporte de materiais com baixo valor agregado em longas distâncias e em grandes quantidades ser muito mais vantajoso por ferrovias do que por rodovias, $60 \%$ dos materiais no país ainda são escoados através de rodovias [3]. Um dos possíveis motivos para explicar tal situação, é a precariedade aliado à baixa eficiência do transporte ferroviário para atender às necessidades dos produtores nacionais.

Tabela 3 - Resultados das interaçóes para a malha.

\begin{tabular}{|c|c|c|c|c|c|c|c|}
\hline Origem & Destino & Distância & Tempo & Origem & Destino & Distância & Tempo \\
\hline Anápolis & Santos & 1188,6 & 85,4 & Rondonópolis & Santos & 1505,0 & 45,3 \\
\hline Anápolis & Itaguaí & 1712,2 & 91,1 & Rondonópolis & Itaguaí & 1873,3 & 71,5 \\
\hline Anápolis & Rio de Janeiro & 1714,2 & 92,8 & Rondonópolis & Rio de Janeiro & 1875,3 & 73,2 \\
\hline Anápolis & Vitória & 1986,0 & 89,6 & Rondonópolis & Vitória & 3034,0 & 104,6 \\
\hline Anápolis & Aracaju & 3092,0 & 185,4 & Rondonópolis & Aracaju & 4140,0 & 200,4 \\
\hline Brasília & Santos & 1268,8 & 84,9 & Bauru & Santos & 466,8 & 24,3 \\
\hline Brasília & Itaguaí & 1792,4 & 90,6 & Bauru & Itaguaí & 835,1 & 50,5 \\
\hline Brasília & Rio de Janeiro & 1794,4 & 92,3 & Bauru & Rio de Janeiro & 837,1 & 52,2 \\
\hline Brasília & Vitória & 2066,2 & 89,1 & Bauru & Vitória & 1995,8 & 83,6 \\
\hline Brasília & Aracaju & 3172,2 & 184,9 & Bauru & Aracaju & 3101,8 & 179,4 \\
\hline
\end{tabular}

Fonte: Os autores (2017).

Por outra perspectiva, sob um olhar quanto à eficiência dos trechos inseridos, alguns pontos se destacaram perante os demais. Isso ocorre devido a que algumas trajetórias, mesmo que com menores distâncias, apresentavam uma maior duração no deslocamento frente a alternativas que, em primeira análise, apresentavam menor eficiência. Em mais de uma interação das matrizes de cada um dos produtos foi indicada a presença de caminhos mínimos divergentes, se comparados com os tempos mínimos de trajeto. Por isso, para um melhor entendimento, as possíveis condiçóes em que se justificariam uma menor eficácia foram levantadas.

Como ponto notável, o trecho Jundiaí - São Paulo apresentou grande importância, devido à sua localidade central e proximidade com o Porto de Santos. Mesmo com uma menor distância entre os nós e confrontando-se com as alternativas (se comparados quanto à duração do trajeto), o trecho em questão possui uma menor eficiência no transporte. Em análise para possível entendimento dos motivos de tal diferença, a localização da parte da linha em região urbanizada pode ser levada em 
consideração, sendo apresentados entraves como passagens de nível críticas e ainda invasôes da faixa de domínio, forçando a redução da velocidade no deslocamento sobre o trecho, conforme indica [4]. Importante observação quando se considera que, sob a esfera do estudo logístico, um maior tempo representa também maiores custos para o transporte de cargas.

Outro trecho analisado que gerou valores significativos para a consideração das redes em geral foi o que tem por destino Aracaju. Este caminho, segundo as interaçôes obtidas pelo Solver, indicou uma menor movimentação de produtos além de maior tempo e distância frente à otimização. Este fato pode ser justificado pela falta de manutençáo das linhas e também pela carência de terminais ao longo desta trajetória, sendo indicada uma menor representatividade deste trajeto.

Ainda, em observação dos resultados, notou-se uma maior eficiência de trajeto da linha Brasília - Santos se comparado com Anápolis - Santos, devido à maior velocidade obtida no primeiro trecho, mas isso pode ser explicado por uma melhor conservação da via perante o segundo trajeto indicado. Por isso, mesmo com uma menor distância para o trecho saindo de Anápolis, a origem de Brasília apresenta menor tempo de duração do transporte para chegar em qualquer um dos destinos de portos (não somente para o caminho mínimo que foi indicado para o destino do porto de Santos).

Apesar de menores distâncias ocorrerem quando os caminhos se destinavam a Santos, as análises indicaram outros fatores importantes relacionadas ao tempo de duração do transporte. Isso pode ser observado, por exemplo, nos caminhos que possuem como destino final o trecho de Vitória, o qual mostrou um tempo de deslocamento muito próximo ao encontrado pelo escoamento ao porto de Santos. Isso pode ser explicado pela observaçáo de que, mesmo com elevada distância, o trecho Belo Horizonte - Vitória possui menores interferências quanto a baldeações ou menores trechos com compartilhamentos de via. Por este motivo, foi contabilizado um menor tempo de espera em terminais ou em passagens críticas das linhas férreas, aumentando a eficiência logística do caminho em que tal trecho pertence.

Entre percursos com similares distâncias, a comparação dos que possuem rumo aos portos do estado do Rio de Janeiro (representados pelos nós de Itaguaí e Rio de Janeiro) implicou em entraves opercionais relevantes a serem considerados. A diferença entre os caminhos mínimos apresentados pelas interaçôes das matrizes dos trechos comentados é de somente dois quilômetros, e os tempos também se assemelham com pouca diferença. Entretanto, quando a tabela dos quantitativos de materiais por trecho é observada, verifica-se que o trajeto com destino a Itaguaí apresenta maior porção dos produtos transportados. Ainda, com um menor tempo e maior valor por quilômetro, é possível observar a grande importância deste último caminho quando se trata do estudo das redes.

\section{Conclusóes}

Com base no conteúdo apresentado, foram obtidos resultados que podem indicar a orientação de novos investimentos a fim de aprimorar e otimizar as malhas ferroviárias existentes no Brasil. 
Para tanto, realizados os estudos com enfoque em Pesquisa Operacional, conseguiu-se identificar menores distâncias e tempo de transporte para a percepção dos gargalos logísticos existentes. Como resultado, foram identificados os caminhos mínimos de Anápolis - Santos e Bauru - Santos (devido à disposição geográfica), ao passo que Brasília - Vitória possui duração muito próxima se comparado com o trecho partindo de Anápolis. Confrontando os resultados obtidos com a base de dados dos quantitativos de materiais transportados ao longo das malhas, pode-se perceber a maior utilizaçáo de determinados trechos para produtos específicos.

Ao se tratar das redes destacadas, divididas pelos produtos (combustíveis, soja, produção agrícola e minério de ferro), alguns pontos obtiveram maior importância, devido a suas características ou relevância para a análise global do estudo apresentado. Por este fato, um dos trechos destacáveis foi o trecho Jundiaí - São Paulo, que quando observado comparativamente ao outro trajeto com destino a Santos, dado por Alumínio - Santos, encontra-se com uma distância menor (cerca de $40 \mathrm{Km}$ ). Entretanto, ao tratar-se de tempo de duração do percurso, o trecho concomitante a este apresenta uma menor duração do que o trajeto passando por Jundiaí, indicando a falta de investimentos de manutenção e ainda a possível problemática encontrada em outros trechos do modal ferroviário brasileiro tais como o compartilhamento de vias, passagens em nível críticas (interferência pelo fluxo de veículos), invasôes de faixa de domínio, aliados à falta de investimentos para a manutenção das vias.

Além deste, outros trechos apresentaram indicativos similares, tais como o trecho Belo Horizonte - Vitória que, mesmo com uma extensão de percurso muito maior que outros trechos pertencentes a outros caminhos mínimos diferentes, apresentou um tempo de duração bem próximo aos demais, superando até mesmo outros trajetos com menores distâncias. Fato que pode ser justificado devido a um menor tempo de espera pelo compartilhamento de via e melhor manutenção desta, dada a elevada importância no transporte do minério de ferro.

Por fim, em trechos que apresentaram distâncias parecidas, como os de origem em Japeri e destinados a Itaguaí ou Rio de Janeiro, foi possível notar a influência da maior quantidade do transporte de carga para a concentração dos investimentos em manutenção de vias. Segundo as informaçóes levantadas, o trecho Japeri - Itaguaí possui um tempo de transporte menor (em torno de duas horas) se comparado ao Japeri - Rio de Janeiro, enquanto suas distâncias diferem em apenas dois quilômetros.

A partir deste trabalho, a análise com base na Pesquisa Operacional proporcionou os conhecimentos necessários para a obtenção dos resultados, mesmo que de forma limitada. Assim, de acordo com esta análise, os pontos notáveis, com a maior necessidade de destinação de investimentos imediatos são: manutenção e duplicação do trecho Jundiaí - São Paulo; conservação e maior desenvolvimento da via do trecho Japeri - Itaguai; destinação de maior quantidade dos produtos para o trecho Belo Horizonte - Vitória; conservação e destinação de produtos transportados para linhas com maior excelência como Alumínio - Santos. Somente proporcionando melhores condiçóes das vias e, portanto, uma maior quantidade de capital também para novos investimentos será possível suprir o gargalo logístico que hoje dificulta que o Brasil se torne mais competitivo perante outros mercados. 


\section{Referências}

[1] ANTF. Informaçóes gerais. Disponível em: <http://www.antf.org.br/informacoes-gerais/>. Acesso em: 7 nov. 2017.

[2] BRONSON, R.; NAADIMUTHU, G. Operations Research, 2nd Ed.. New York:, 1997.

[3] CNT. Modal Rodoviário. Disponível em: <http://www.cnt.org.br/Modal/modal-rodoviario-cnt>. Acesso em: 7 nov. 2017.

[4] CNT, Pesquisa CNT de ferrovias. [S.L.: s.n.], 2015.

[5] DANTZIG, George B.. Linear Programming and Extensions. United States: [s.n.], 1963.

[6] MARÓTI, Gábor. Operations research models for railway rolling stock planning. Eindhoven: Technische Universiteit Eindhoven, 2006.

[7] SANTOS, Mauricio Pereira Dos. Programaçáo linear. [S.L.]: Universidade do Estado do Rio de Janeiro, 2007. 